\title{
Microscopic Theory for the Quantum to Classical Crossover in Chaotic Transport
}

\author{
Robert S. Whitney ${ }^{1}$ and $\mathrm{Ph}$. Jacquod ${ }^{1}$ \\ ${ }^{1}$ Département de Physique Théorique, Université de Genève, CH-1211 Genève 4, Switzerland
}

(Dated: August 23, 2004)

\begin{abstract}
We present a semiclassical theory for the scattering matrix $\mathcal{S}$ of a chaotic ballistic cavity at finite Ehrenfest time. Using a phase-space representation coupled with a multi-bounce expansion, we show how the Liouville conservation of phase-space volume decomposes $\mathcal{S}$ as $\mathcal{S}=\mathcal{S}^{\mathrm{cl}} \oplus \mathcal{S}^{\mathrm{qm}}$. The short-time, classical contribution $\mathcal{S}^{\mathrm{cl}}$ generates deterministic transmission eigenvalues $T=0$ or 1 , while quantum ergodicity is recovered within the subspace corresponding to the long-time, stochastic contribution $\mathcal{S}^{\mathrm{qm}}$. This provides a microscopic foundation for the two-phase fluid model, in which the cavity acts like a classical and a quantum cavity in parallel, and explains recent numerical data showing the breakdown of universality in quantum chaotic transport in the deep semiclassical limit. We show that the Fano factor of the shot-noise power vanishes in this limit, while weak localization remains universal.
\end{abstract}

PACS numbers: 73.23.-b, 74.40.+k, 05.45.Mt

Quantum mechanics strongly alters the classical theory of transport. In mesoscopic systems, spectacular new phenomena of purely quantum origin emerge, such as weak localization, universal conductance fluctuations [1], and sub-Poissonian shot-noise 2]. In both mesoscopic disordered diffusive metals and clean chaotic ballistic systems, these phenomena have been found to exhibit a universality well captured by Random Matrix Theory (RMT) 3]. For instance, in a time-reversal-symmetric chaotic cavity, RMT correctly predicts that weak localization reduces the conductance below its classical value by $\delta g_{\mathrm{rmt}}=-1 / 4$, that the variance of the conductance $\sigma_{\text {rmt }}^{2}(g)=1 / 8$ (conductances are expressed in units of $\left.e^{2} / h\right)$, and that the shot-noise power is reduced by the Fano factor $F_{\mathrm{rmt}}=1 / 4$, below the Poissonian value of $2 e\langle I\rangle,\langle I\rangle$ being the average current through the sample.

Despite the many successes of RMT it has become clear, following the work of Aleiner and Larkin [4], that universality is broken for those quantum chaotic systems in which the Ehrenfest time becomes a relevant time scale. The Ehrenfest time, $\tau_{\mathrm{E}}$, corresponds to the time it takes for a minimal wavepacket to be stretched over a length comparable to a classical characteristic length of the system. For a closed chaotic cavity of linear size $L$, the Ehrenfest time is $\tau_{\mathrm{E}}^{\mathrm{c}} \simeq \lambda^{-1} \ln \left[L / \lambda_{\mathrm{F}}\right]$, in term of the classical cavity's Lyapunov exponent $\lambda$ and the Fermi wavelength $\lambda_{\mathrm{F}}[5]$. For the same cavity attached to twoleads of width $W$ there is a second shorter Ehrenfest time $\tau_{\mathrm{E}}^{\mathrm{o}}=\tau_{\mathrm{E}}^{\mathrm{c}}-2 \lambda^{-1} \ln [L / W][\underline{6}]$. These two quantum mechanical time scales differ only by a classical quantity. For times shorter than the Ehrenfest time, the quantum mechanical time evolution of a narrow wavepacket is given by the solution of classical equations of motion [5, 7], thus strong deviations from the RMT of transport emerge once $\tau_{\mathrm{E}}^{\mathrm{O}}$ is no longer negligible. It is for example well established that, as $\tau_{\mathrm{E}}^{\mathrm{o}, \mathrm{c}} \rightarrow \infty$, the Fano factor disappears [8, 9, 10, 11] and parametric conductance fluctuations remain universal while sample-to-sample conductance fluctuations do not 11, 12. However the analytical prediction of Refs. [4, 13] that weak localization corrections also disappear has recently been challenged numerically [14].

These observations are all consistent with the phenomenological two-phase fluid model [1, 15. In this model, introduced in Ref. 15], it is assumed that electrons with short dwell time $<\tau_{\mathrm{E}}^{\mathrm{o}}$ are transmitted deterministically through the cavity, while quantum mechanical stochasticity is recovered for those with longer dwell time $>\tau_{\mathrm{E}}^{\mathrm{o}}$. Accordingly, the system splits into two cavities in parallel; a classical, deterministic cavity, with an effective number of modes $N_{\mathrm{cl}}=N\left(1-\exp \left[-\tau_{\mathrm{E}}^{\mathrm{o}} / \tau_{\mathrm{D}}\right]\right)$, and a quantum mechanical, stochastic cavity having $N_{\mathrm{qm}}=N \exp \left[-\tau_{\mathrm{E}}^{\mathrm{o}} / \tau_{\mathrm{D}}\right]$ modes $\left(\tau_{\mathrm{D}}\right.$ is the average dwell time). With the further assumption that the stochastic cavity obeys RMT [1, 15], the observed numerical behavior of shot-noise and of conductance fluctuations follows 10, 11, 12]. The universal value of parametric conductance fluctuations is maintained as long as $N_{\mathrm{qm}} \propto \hbar_{\text {eff }}^{-1+1 / \lambda \tau_{\mathrm{D}}} \gg 1$, where the effective Planck's constant $\hbar_{\text {eff }} \equiv \lambda_{F} / L\left(\lambda_{F}\right.$ is the Fermi wavelength). For fully developed chaotic dynamics $\lambda \tau_{\mathrm{D}} \gg 1$, and so this condition for $N_{\mathrm{qm}}$ is always satisfied in the semiclassical limit of $\hbar_{\mathrm{eff}} \rightarrow 0$ 11. However the prediction that weak localization remains universal (independently of $\left.\hbar_{\text {eff }}\right)$ is in direct contradiction of the analytical prediction $\delta g \propto \exp \left[-\tau_{\mathrm{E}}^{\mathrm{c}} / \tau_{\mathrm{D}}\right]$ of Refs. [4, 13]. Both this controversy and the successes of the phenomenological two-phase fluid model call for a microscopic foundation of this model. The purpose of this letter is to provide such a foundation.

We consider an open, two-dimensional chaotic quantum dot, ideally connected to two external leads, each carrying $N \gg 1$ modes. We require that the size of the openings to the leads is much smaller than the perimeter of the cavity but is still semiclassically large, $1 \ll N \ll L / \lambda_{F}$. This ensures that the chaotic dynamics inside the dot has enough time to develop, i.e. $\lambda \tau_{\mathrm{D}} \gg 1$. The classical dynamics within the cavity can be described by its Birkhoff map, which provides a recursive relation for the transverse momentum $p_{\tau}$ and position $q_{\tau}$ of incidence at the $\tau^{\text {th }}$ collision with the cavity's boundary. 
Quantizing this map leads to a unitary, $M \times M$ Floquet operator $\mathcal{V}$ where $M=\operatorname{Int}\left[\hbar_{\text {eff }}^{-1}\right]$. A particle initially in a state $\psi_{0}$ occupies a state $\psi_{\tau}=\mathcal{V}^{\tau} \psi_{0}$ after $\tau$ collisions at the cavity's boundary. The transport properties of this system derive from its scattering matrix [16]

$$
\mathcal{S}=\left(\begin{array}{ll}
\mathbf{r} & \mathbf{t}^{\prime} \\
\mathbf{t} & \mathbf{r}^{\prime}
\end{array}\right)
$$

which we write in terms of $N \times N$ transmission (t) and reflection $(\mathbf{r})$ matrices. From $\mathcal{S}$, the system's conductance is given by $g=\operatorname{Tr}\left(\mathbf{t}^{\dagger} \mathbf{t}\right)$. To construct $\mathcal{S}$ from $\mathcal{V}$, we couple the cavity to the left (L) and right $(\mathrm{R})$ leads by introducing a $2 N \times M$ projection matrix $P=P^{(\mathrm{L})}+P^{(\mathrm{R})}$. Expressed in the basis of channel modes, the projection matrices read $P_{n m}^{(\mathrm{L}, \mathrm{R})}=1$ if $m=n \in\left\{m_{i}^{(\mathrm{L}, \mathrm{R})}\right\}$ and $P_{n m}^{(\mathrm{L}, \mathrm{R})}=0$ otherwise. The sets $\left\{m_{i}^{(\mathrm{L}, \mathrm{R})}\right\}$ are the $N$ components of $\mathcal{V}$ ideally connected to the modes of the $\mathrm{L}$ or $\mathrm{R}$ lead respectively. The energy-dependent $\mathcal{S}$ becomes 17.

$$
\begin{aligned}
\mathcal{S}(\varepsilon) & =\sum_{\tau=0}^{\infty} \exp [i(\tau+1) \varepsilon] \mathcal{S}_{\tau}, \\
\mathcal{S}_{\tau}(\varepsilon) & =P\left[\mathcal{V}\left(1-P^{T} P\right)\right]^{\tau} \mathcal{V} P^{T}
\end{aligned}
$$

The $\tau^{\text {th }}$ term in the above Taylor expansion corresponds to the time-evolution operator for a particle colliding exactly $\tau$ times at the boundary of the cavity before exiting.

To maximally resolve the underlying classical dynamics, we wish to express $\mathcal{S}$ in an appropriate orthonormal basis. We start from a complete set of coherent states centered on a discrete von Neumann lattice 18. While this set is not orthogonal, it is nevertheless complete (and not overcomplete). It is then possible to recursively construct an orthonormal Phase Space (PS) basis from it, $\left\{\left|(p, q)_{i}\right\rangle_{\mathrm{L}, \mathrm{R}}\right\}$, where each PS basis state is exponentially localized in both position and momentum and covers a phase-space area $\mathcal{O}(\hbar)$ 20. The algorithm for constructing this basis is similar to those used in wavelet analysis [19], and we will discuss it elsewhere 21]. Here we only show such a PS state in Fig. [1 The transformation from lead modes to this orthonormal basis is a unitary transformation $\mathcal{S} \rightarrow \mathcal{U}^{\dagger} \mathcal{S U}$ and hence leaves the transport properties of $\mathcal{S}$ unaffectedx. We will now show that the multi-bounce expansion of Eqs. (2), expressed in this orthonormal PS basis elegantly connects quantum transport to the underlying classical dynamics.

Two cases have to be considered when evaluating the matrix elements $\left\langle(p, q)_{i}\left|\mathcal{S}_{\tau}\right|(p, q)_{j}\right\rangle$ of $\mathcal{S}_{\tau}$. If $\left|(p, q)_{j}\right\rangle$ sits on classical trajectories which all leave the cavity at the same time and through the same lead, then its quantum mechanical time-evolution is given by the classical one. This requires first that the classical trajectory starting at $(p, q)_{j}$ leaves the cavity after a time $\tau<\tau_{\mathrm{E}}^{\mathrm{O}}[\underline{5}]$ (this ensures that $\mathcal{S}_{\tau}\left|(p, q)_{j}\right\rangle$ is still a well localized wavepacket), and second that at the time of escape the wavepacket is not located too close to the edge of the lead, and so

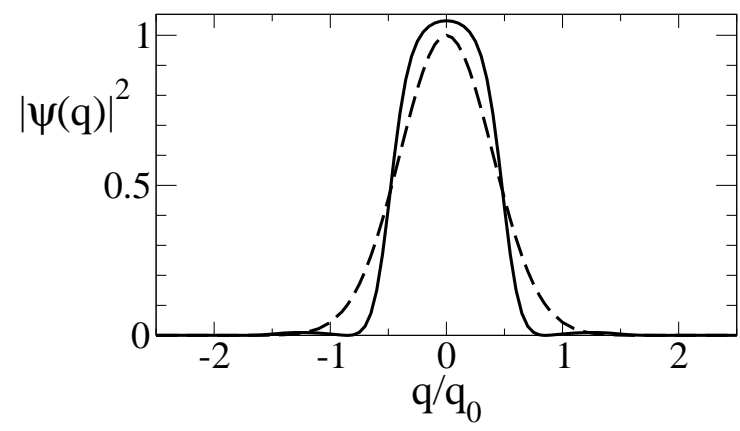

FIG. 1: Squared amplitude of a PS state (solid line) versus the normalized coordinate $q / q_{0}, q_{0}=L \sqrt{2 \pi \hbar_{\mathrm{eff}}}$. For comparison we also show a coherent state (dashed line).

avoids partial reflection. When these two conditions are fulfilled, the whole wavepacket escapes the cavity at a single time $\tau$ through either the L or R lead (not both) in a state $\mathcal{S}_{\tau}\left|(p, q)_{j}\right\rangle$. In all other instances this is not the case. For wavepackets that exit the cavity either at times $\tau>\tau_{\mathrm{E}}^{\mathrm{o}}$ or after one or more partial reflections at the edge of a lead, diffraction and quantum coherence play an important role. Thus we split $\mathcal{S}$ into a shortand a long-time contribution [22],

$$
\begin{aligned}
&\left\langle(p, q)_{i}|\mathcal{S}|(p, q)_{j}\right\rangle=\left\langle(p, q)_{i}\left|\mathcal{S}^{\mathrm{cl}}+\mathcal{S}^{\mathrm{qm}}\right|(p, q)_{j}\right\rangle, \\
& \mathcal{S}^{\mathrm{cl}}=\sum_{\tau<\tau_{\mathrm{E}}^{\mathrm{o}}} \mathcal{S}_{\tau} \quad ; \quad \mathcal{S}^{\mathrm{qm}}=\sum_{\tau>\tau_{\mathrm{E}}^{\mathrm{o}}} \mathcal{S}_{\tau} .
\end{aligned}
$$

The classical Liouville conservation of PS volume guarantees that $\mathcal{S}^{\mathrm{cl}}$ and $\mathcal{S}^{\mathrm{qm}}$ act on different, nonoverlapping subsets of the PS basis. To see this, we recall that the evolution of wavepackets that are initially localized in both position and momentum is very well approximated semiclassically as a sum over classical trajectories [7, 23]. Thus if a classical trajectory contributes to $\mathcal{S}^{\text {cl }}$, then it does not contribute to $\mathcal{S}^{\mathrm{qm}}$. From this $L i$ ouville blocking, we conclude that the PS states divide into two non-overlapping subsets (up to exponentially small corrections) (i) the fully classical ones which leave the cavity after short times $\tau<\tau_{\mathrm{E}}^{\mathrm{o}}$ and are transmitted or reflected deterministically, and (ii) the quantum ones which stay inside the cavity for longer times $\tau>\tau_{\mathrm{E}}^{\mathrm{o}}$ and/or are partially reflected and transmitted at collision at the edge of a lead 24]. From the dwell time distribution $\rho(\tau)=\tau_{\mathrm{D}}^{-1} \exp \left[-\tau / \tau_{\mathrm{D}}\right]$ in chaotic cavities, one gets a number $N_{\mathrm{cl}}=N\left(1-\exp \left[-\tau_{\mathrm{E}}^{\mathrm{o}} / \tau_{\mathrm{D}}\right]\right)$ of states in subset (i) and $N_{\mathrm{qm}}=N \exp \left[-\tau_{\mathrm{E}}^{\mathrm{o}} / \tau_{\mathrm{D}}\right] \propto \hbar_{\mathrm{eff}}^{-1+1 / \lambda \tau_{\mathrm{D}}} \gg 1$ of states in subset (ii). These considerations lead to our first result that $\mathcal{S}$ has the block-diagonal decomposition

$$
\mathcal{S}=\mathcal{S}^{\mathrm{cl}} \oplus \mathcal{S}^{\mathrm{qm}},
$$

where $\mathcal{S}^{\mathrm{cl}, \mathrm{qm}}$ both have the structure given in Eq. (11). Thus the transmission matrix $\mathbf{T}=\mathbf{t}^{\dagger} \mathbf{t}$ is also blockdiagonal

$$
\mathbf{T}=\mathbf{T}^{\mathrm{cl}} \oplus \mathbf{T}^{\mathrm{qm}},
$$


with $N_{\mathrm{cl}, \mathrm{qm}}$ by $N_{\mathrm{cl}, \mathrm{qm}}$ matrices $\mathbf{T}^{\mathrm{cl}, \mathrm{qm}}=\left(\mathbf{t}^{\mathrm{cl}, \mathrm{qm}}\right)^{\dagger} \mathbf{t}^{\mathrm{cl}, \mathrm{qm}}$. Accordingly, the system's conductance is given by the sum of two contributions

$$
g=g_{\mathrm{cl}}+g_{\mathrm{qm}},
$$

where $g_{\mathrm{cl}}=N_{\mathrm{cl}} / 2$ and $g_{\mathrm{qm}} \simeq N_{\mathrm{qm}} / 2$. Note that $g_{\mathrm{qm}}$ gives the contribution to the total conductance carried by the stochastic scattering states. As we will see below, it contains the weak localization corrections. Eqs. (46) give a microscopic justification of the two-phase fluid model previously postulated in Refs. 11, 15], where the finite $\tau_{\mathrm{E}}^{\mathrm{o}, \mathrm{c}}$ transport properties of a quantum chaotic system are given by those of two separated cavities put in parallel. The decomposition (4) of $\mathcal{S}$ is naturally connected to the underlying classical dynamics. In fact, because of the finiteness of $\tau_{\mathrm{D}}$, classical trajectories injected into a cavity are naturally grouped into PS transmission and reflection bands [15], despite the ergodicity of the associated closed cavity. These bands are best visualized by considering PS cross-sections of the left and right leads, and plotting the initial and final coordinates of trajectories transmitted from left to right. This is sketched in Fig. 2. All trajectories within one band follow neighboring paths and exit at the same time $\tau$ through the same lead. Because of the chaotic classical dynamics, bands with longer escape times $\tau$ are narrower, having a width (and hence a PS area) scaling like $\propto \exp [-\lambda \tau]$. The Ehrenfest time is the time at which this area becomes smaller than $\hbar_{\mathrm{eff}}$, consequently for times longer than $\tau_{\mathrm{E}}^{\mathrm{o}}$ no band is big enough for a PS state to fit within it 15 . The meaning of Eq. (4) is now obvious: The matrix $\mathcal{S}^{\mathrm{cl}}$ acts in the subspace of PS states which are entirely inside a single band, while $\mathcal{S}^{\mathrm{qm}}$ acts in the subspace of PS states which overlap many bands, exiting at different times, and in general through different leads.

We now discuss the properties of $\mathbf{T}^{\mathrm{cl}}$ and $\mathbf{T}^{\mathrm{qm}}$. The classical part of the transmission matrix is $\sum_{\tau, \tau^{\prime}<\tau_{\mathrm{E}}^{\mathrm{o}}} \mathbf{t}_{\tau}^{\dagger} \mathbf{t}_{\tau^{\prime}}$. However each PS state in $\mathbf{t}^{\mathrm{cl}}$ follows a band that exits at a well defined time, thus only diagonal terms in the double sum over $\tau, \tau^{\prime}$ are non-zero, and one has

$$
\mathbf{T}^{\mathrm{cl}}=\sum_{\tau<\tau_{\mathrm{E}}^{\mathrm{O}}} \mathbf{t}_{\tau}^{\dagger} \mathbf{t}_{\tau}
$$

We stress that this is not the usual diagonal approximation and so we do not require any energy or ensemble averaging here. Once $\mathbf{T}^{\mathrm{cl}}$ is written as in Eq. (7), it is easy to show that it is diagonal. To see this one recalls first that for $\tau<\tau_{\mathrm{E}}^{\mathrm{o}}, \mathcal{S}_{\tau}\left|(p, q)_{j}\right\rangle=\left|(\tilde{p}, \tilde{q})_{i}\right\rangle$ is still a narrow wavepacket, which however has been stretched and rotated by the classical dynamics [5] (this is sketched in Fig. (2). This can be included for each band individually into a $\tau$-dependent area-preserving coordinate transformation, so that a basis made of these stretched PS states is complete and orthonormal within each band (relationships between bands can be ignored because Liouville blocking ensures that bands never overlap). This

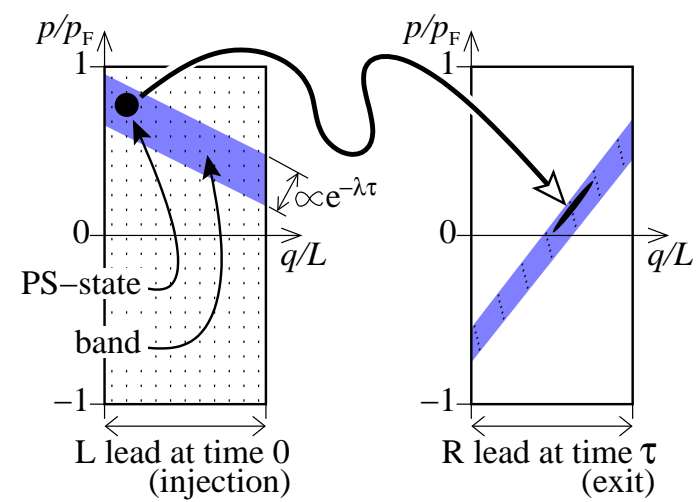

FIG. 2: (color online) Phase space of the injection (Left) and exit (Right) leads. The shaded area depicts a classical transmission band which goes from $\mathrm{L}$ to $\mathrm{R}$ in time $\tau$. Superimposed on the $\mathrm{L}$ lead is a square von Neumann lattice with spacing $\left(2 \pi \hbar_{\mathrm{eff}}\right)^{1 / 2}$. A PS state is placed at every vertex on the lattice, forming a complete orthonormal basis. Each PS state initially covers a circle of radius $\mathcal{O}\left[\hbar_{\text {eff }}^{1 / 2}\right]$ in phase space. For $\tau<\tau_{\mathrm{E}}^{\mathrm{O}}$, this state is still localized, but is stretched under the dynamics, as indicated by the dark ellipse on the $\mathrm{R}$ lead.

means that, after a $\tau$-dependent unitary transformation of the bra-states, $\left\langle(\tilde{p}, \tilde{q})_{i}\left|\mathbf{t}_{\tau}\right|(p, q)_{j}\right\rangle=0$ unless the state $\left|(p, q)_{j}\right\rangle$ in the $\mathrm{L}$ lead is the one state that transmits to the (stretched and rotated) state $\left|(\tilde{p}, \tilde{q})_{i}\right\rangle$ in the $\mathrm{R}$ lead, then $\left\langle(\tilde{p}, \tilde{q})_{i}\left|\mathbf{t}_{\tau}\right|(p, q)_{j}\right\rangle=\exp \left[i \varphi_{i j}\right]$. Eq. (7) shows that $\mathbf{T}^{\mathrm{cl}}$ is unaffected by this transformation, hence

$$
\mathbf{T}_{i j}^{\mathrm{cl}}=\sum_{\tau<\tau_{\mathrm{E}}^{\mathrm{O}}}\left|\left[\mathbf{t}_{\tau}\right]_{i j}\right|^{2}=\delta_{i j} \times T_{i}, \quad i \in\left\{1, \cdots N_{\mathrm{cl}}\right\},
$$

with transmission eigenvalues $T_{i}$ which are either zero or one. While this result was anticipated [25], to the best of our knowledge it is derived here for the first time directly from a microscopic theory. Thus $\mathbf{T}^{\mathrm{cl}}$ cannot contribute to noise, moreover, all quantum phases (such as $\varphi_{i j}$ ) cancel in $\mathbf{T}^{\mathrm{cl}}$ and hence it carries no quantum coherence. Both shot-noise and coherent effects like weak localization are carried solely by $\mathbf{T}^{\mathrm{qm}}$ which we now focus on.

Unlike in $\mathbf{T}^{\mathrm{cl}}$, the off-diagonal terms $\tau \neq \tau^{\prime}$ strongly influence $\mathbf{T}^{\mathrm{qm}}=\sum_{\tau, \tau^{\prime}>\tau_{\mathrm{E}}^{\mathrm{o}}} \mathbf{t}_{\tau}^{\dagger} \mathbf{t}_{\tau^{\prime}}$. These terms give rise to coherence and to the pseudo-randomness of transmission phases, as many initial PS states may now be partially transferred to the same final PS state. Conversely, each initial PS state is partially transferred to many final PS states [5]. Taking the Liouville blocking into account, $\mathbf{t}^{\mathrm{qm}}$ is ergodic within its own subspace (being decoupled from $\mathbf{t}^{\mathrm{cl}}$ ). Because it is made up of all the longtime contributions with $\tau>\tau_{\mathrm{E}}^{\mathrm{o}}$, it contains dynamically diffractive contributions necessary for weak localization (Richter-Sieber pairs [26]). We further note that its size $N_{\mathrm{qm}} \propto \hbar_{\mathrm{eff}}^{-1+1 / \lambda \tau_{\mathrm{D}}} \rightarrow \infty$ in the semiclassical limit.

This is all we need to generalize the semiclassical theory of weak localization to finite $\tau_{\mathrm{E}}^{\mathrm{o}, \mathrm{c}} / \tau_{\mathrm{D}}$. The approach of Ref. 26] can be followed provided Eq. (4) is taken into account by considering an effective cavity with a 
number of modes $N_{\mathrm{qm}}$, a restricted phase-space area $\Sigma_{\mathrm{qm}}(E)=\Sigma(E) \exp \left[-\tau_{\mathrm{E}}^{\mathrm{o}} / \tau_{\mathrm{D}}\right]$ and a dwell time distribution $\rho_{\mathrm{qm}}(\tau)=\Theta\left(\tau-\tau_{\mathrm{E}}^{\mathrm{o}}\right) \exp \left[-\left(\tau-\tau_{\mathrm{E}}^{\mathrm{o}}\right) / \tau_{\mathrm{D}}\right] / \tau_{\mathrm{D}} . \quad \mathrm{A}$ careful analysis of the dwell time for the Richter-Sieber pairs (and use of an optimal basis 20]) yields $\delta g=$ $-1 / 4\left(1+\mathcal{O}\left[\left(\lambda \tau_{\mathrm{D}}\right)^{-1}\right]\right)$ independent of $\tau_{\mathrm{E}}^{\mathrm{O}, \mathrm{c}}[27]$. Thus for good chaotic systems, $\lambda \tau_{\mathrm{D}} \gg 1$, weak localization remains universal in the classical limit.

Turning our attention to shot-noise, we readily see that the reduction of the fraction of quantum channels reproduces the predicted exponential suppression of the Fano factor in the large $\tau_{\mathrm{E}}^{\mathrm{o}}$ regime [8], $F=\sum_{n} T_{n}(1-$ $\left.T_{n}\right) / \sum_{n} T_{n} \propto N_{\mathrm{qm}} / N=\exp \left[-\tau_{\mathrm{E}}^{\mathrm{o}} / \tau_{\mathrm{D}}\right]$. We expect there will soon be a semiclassical theory to explain the universal value $F=1 / 4$. Indeed we believe that a theory of transmission along the lines of Ref. 28] is possible, which would show that the properties of $\mathcal{S}^{\mathrm{qm}}$ are captured by one of the circular ensembles of random matrices. Assuming that this is the case, we have the full distribution $P(T)$ of transmission eigenvalues $P(T)=$ $\alpha P_{\text {rmt }}(T)+(1-\alpha)[\delta(T)+\delta(1-T)] / 2$, where $P_{\text {rmt }}(T)=$
$\pi^{-1}[T(1-T)]^{-1 / 2}[3]$ and $\alpha=\exp \left[-\tau_{\mathrm{E}}^{\mathrm{o}} / \tau_{\mathrm{D}}\right]$, as postulated in Ref. 15] and numerically observed in Ref. 11]. If confirmed analytically, this would give the full counting statistics of the current for any value of $\tau_{\mathrm{E}}^{\mathrm{o}} / \tau_{\mathrm{D}}$, including the correct Fano factor $F=(1 / 4) \exp \left[-\tau_{\mathrm{E}}^{\mathrm{o}} / \tau_{\mathrm{D}}\right]$ [8, 29].

In summary, we have constructed a theory for transport in quantum chaotic ballistic systems in the regime of finite $\tau_{\mathrm{E}}^{\mathrm{o}, \mathrm{c}} / \tau_{\mathrm{D}}$. Our theory confirms the separation of the system into two subsystems, and thus provides a microscopic foundation for the two-phase fluid model [11, 15]. Weak localization is predicted to remain universal in lowest order in $1 / \lambda \tau_{\mathrm{D}}$, for any $\hbar_{\mathrm{eff}}$, in agreement with numerical data 14], but in contradiction with earlier theories [4, 13. Whether $\mathcal{S}^{\mathrm{qm}}$ is a random matrix remains an open question.

We are grateful to E. Sukhorukov for very interesting discussions and thank I. Adagideli, C. Beenakker, M. Sieber and P. Silverstrov for helpful comments. This work is supported by the Swiss National Science Foundation.
[1] A.D. Stone, in Physics of Nanostructures, J.H. Davies and A.R. Long Eds., Institute of Physics Publishing (1992).

[2] Ya.M. Blanter and M. Büttiker, Phys. Rep. 336, 1 (2000).

[3] C.W.J. Beenakker, Rev. Mod. Phys. 69, 731 (1997).

[4] I.L. Aleiner and A.I. Larkin, Phys. Rev. B 54, 14423 (1996).

[5] G.M. Zaslavsky, Phys. Rep. 80, 157 (1981).

[6] M.G. Vavilov and A.I. Larkin, Phys. Rev. B 67, 115335 (2003).

[7] E.J. Heller, and S. Tomsovic, Phys. Today 46(7), 38 (1993).

[8] O. Agam, I. Aleiner and A. Larkin, Phys. Rev. Lett. 85, 3153 (2000).

[9] S. Oberholzer, E.V. Sukhorukov, and C. Schönenberger, Nature 415, 765 (2002); E.V. Sukhorukov and O.M. Bulashenko, Phys. Rev. Lett. (to be published) cond-mat/0408075

[10] J. Tworzydło, A. Tajic, H. Schomerus, and C.W.J. Beenakker, Phys. Rev. B 68, 115313 (2003).

[11] Ph. Jacquod and E.V. Sukhorukov, Phys. Rev. Lett. 92, 116801 (2004).

[12] J. Tworzydło, A. Tajic, and C.W.J. Beenakker, Phys. Rev. B 69, 165318 (2004).

[13] İ. Adagideli, Phys. Rev. B 68, 233308 (2003).

[14] J. Tworzydło, A. Tajic, and C.W.J. Beenakker, Phys. Rev. B 70, 205324 (2004).

[15] P.G. Silvestrov, M.C. Goorden, and C.W.J. Beenakker, Phys. Rev. B 67, 241301(R) (2003).

[16] M. Büttiker, IBM J. Res. Dev. 32, 317 (1988).

[17] Y.V. Fyodorov and H.-J. Sommers, JETP Letters 72, 422 (2000).

[18] See e.g. Chap. 1.4 in: A. Perelomov, Generalized Co- herent States and Their Application, (Springer-Verlag, Berlin, 1986).

[19] See e.g.: G. Kaiser, A Friendly Guide to Wavelets (Birkhäuser, Boston, 1994).

[20] This basis (chosen for pedagogical reasons) underestimates $N_{\mathrm{cl}}$. Both this basis and an optimal choice will be discussed at length in Ref. [21].

[21] Ph. Jacquod and R.S. Whitney, in preparation.

[22] In Eqs. (31) it is understood that the $\tau<\tau_{\mathrm{E}}^{\mathrm{o}}$, part of $\mathcal{S}$ that leads to partial reflection at the edge of a lead is included in $\mathcal{S}^{\mathrm{qm}}$. This part has a subdominantly small size $\mathcal{O}\left(\hbar_{\text {eff }}^{-1 / 2}\right) \ll N_{\text {qm }}$.

[23] This is true up to algebraically long times $\tau^{*}=$ $\mathcal{O}\left(\hbar_{\text {eff }}^{-a}\right) \gg \tau_{\mathrm{E}}^{\mathrm{o}, \mathrm{c}}(a>0$, typically $a \approx 1 / 2)[7]$. In the semiclassical limit $\hbar_{\mathrm{eff}} \rightarrow 0$, this approach captures the transport properties arising from all but an exponentially small number of modes $\propto \hbar_{\text {eff }}^{-1} \exp \left[-1 / \hbar_{\text {eff }}^{a} \tau_{\mathrm{D}}\right]$.

[24] The basis transformation from channel modes to PS states can only be made unitary if the PS states which sit on the edge of the leads are replaced by other states which are entirely within the lead. These edge-of-lead states will be diffracted and so are included in $\mathcal{S}^{\mathrm{qm}}$ as in 22]. This will be discussed at length in 21].

[25] C.W.J. Beenakker and H. van Houten, Phys. Rev. B 43, R12066 (1991).

[26] K. Richter and M. Sieber, Phys. Rev. Lett. 89, 206801 (2002).

[27] This result is easily generalized to asymmetric leads [21].

[28] S. Müller, S. Heusler, P. Braun, F. Haake, and A. Altland, Phys. Rev. Lett. 93, 014103 (2004).

[29] Contrary to what is commonly implied, the shot-noise power at fixed bias does not vanish in the limit $\tau_{\mathrm{E}}^{\mathrm{o}} / \tau_{\mathrm{D}} \rightarrow$ $\infty$ since it depends on $N_{\mathrm{qm}}$, and not on $N_{\mathrm{qm}} / N$. 